\title{
Local topological properties of asymptotic cones of groups
}

\author{
GREGORY R CONNER \\ CURTIS KENT
}

\begin{abstract}
We define a local analogue to Gromov's loop division property which we use to give a sufficient condition for an asymptotic cone of a complete geodesic metric space to have uncountable fundamental group. When considering groups our condition allows us to relate the local connectedness properties of the asymptotic cone with combinatorial properties of the group. This is used to understand the asymptotic cones of many groups actively being studied in the literature.
\end{abstract}

20F65; 20F69

Gromov [14, Section 5.F] was first to notice a connection between the homotopic properties of asymptotic cones of a finitely generated group and algorithmic properties of the group; if all asymptotic cones of a finitely generated group are simply connected, then the group is finitely presented, its Dehn function is bounded by a polynomial which implies that its word problem is in NP, and its isodiametric function is linear. A version of that result for higher homotopy groups was proved by Riley [25]. The converse statement does not hold; there are finitely presented groups with nonsimply connected asymptotic cones and polynomial Dehn functions (see Bridson [1] and Sapir, Birget and Rips [26]) and even with polynomial Dehn functions and linear isodiametric functions (see Olshanskii and Sapir [21]). A partial converse statement was proved by Papasoglu [23]: a group with quadratic Dehn function has all asymptotic cones simply connected. For groups with subquadratic Dehn functions (ie hyperbolic groups) the statement was previously proved by Gromov [13]: all asymptotic cones in that case are $\mathbb{R}$-trees. An example of Thomas and Velickovic [28] shows that for a finitely generated group one asymptotic cone can be a tree, and hence simply connected, while another asymptotic cone may have nontrivial fundamental group. In Section 3, we show how to modify Thomas and Velickovic's example to obtain a finitely generated group with one asymptotic cone which is an $\mathbb{R}$-tree and one asymptotic cone which is not locally simply connected. This gives another example of a group with asymptotic cones which are not locally bilipschitz. In Olshanskii, Osin and Sapir [20] a group is constructed with not locally bilipschitz asymptotic cones. Even more, it gives an 
example of a finitely generated group with asymptotic cones which are not locally homotopy equivalent.

If a group is finitely presented and one of its asymptotic cone is an $\mathbb{R}$-tree, then the group is hyperbolic, so all of its asymptotic cones are simply connected (this essentially follows from Gromov's version of the Cartan-Hadamard theorem for hyperbolic groups, see the appendix of [20]). Nevertheless in [22] a finitely presented group which is a multiple HNN extension of a free group having both simply connected and nonsimply connected asymptotic cones was constructed.

In [14] Gromov defined the notion of a loop division property and used it to outline a proof that a metric space has the loop division property if and only if all of its asymptotic cones are simply connected. Papasoglu presented a proof of the only if direction in [23] and Druţu gave a proof of the if direction in [7]. A version of the loop division property which guarantees that a particular asymptotic cone is simply connected was used by Olshanskii and Sapir in [22]. In the current article we will define an analogue to Gromov's loop division property which we use to understand the local topological structure of asymptotic cones.

In Section 2 we recall some of the definitions and consequences of Gromov's loop division property as studied by Papasoglu and define a local version which we call $\epsilon$-coarsely loop divisible which depends on an ultrafilter, $\omega$, and a scaling sequence, $d$. We prove that a space is $\epsilon$-coarsely loop divisible with respect to $(\omega, d)$ if and only if all sufficiently short loops in $\operatorname{Con}^{\omega}(X, e, d)$ can be partitioned into strictly shorter loops where $e$ is an observation sequence. We say that a space is uniformly $\epsilon-$ coarsely divisible if the number of pieces required to partition small loops in $\operatorname{Con}^{\omega}(X, e, d)$ is uniformly bounded independently of the chosen loop. In general an asymptotic cone of a metric space will depend on the choice of scaling sequence, observation sequence and ultrafilter and hence it is natural to define a loop division property which also depends on these choices. For homogeneous spaces, the isometry type of an asymptotic cone does not depend on the choice of base point: when considering homogeneous space we will frequently either drop the base point from our notation or not specify which observation sequence is chosen.

Theorem A (Propositions 2.9, 2.14, 2.10) Suppose $G$ is a finitely generated group, $\omega$ is an ultrafilter on $\mathbb{Z}$ and $d$ is an $\omega$-divergent sequence.

(1) If $G$ is uniformly $\epsilon$-coarsely loop divisible, then $\operatorname{Con}^{\omega}(G, d)$ is uniformly locally simply connected and $G$ has an asymptotic cone which is simply connected.

(2) If $\operatorname{Con}^{\omega}(G, d)$ is semilocally simply connected, then $G$ is $\epsilon$-coarsely loop divisible. 
Papasoglu (see Proposition 2.7) showed that if one requires $G$ to be uniformly $\epsilon-$ coarsely loop divisible with respect to $(\omega, d)$ for every $\epsilon>0$, then one obtains that $\operatorname{Con}^{\omega}(G, d)$ is actually simply connected. It is not clear if uniformly coarsely divisible is actually a necessary condition. Hence the following questions are open.

For the remainder of this section $G$ will denote a finitely presented group.

Question 1 If $\operatorname{Con}^{\omega}(G, d)$ is locally simply connected is $G$ uniformly $\epsilon$-coarsely loop divisible for some $\epsilon$ ?

Question 2 If $\operatorname{Con}^{\omega}(G, d)$ is simply connected is $G$ uniformly $\epsilon$-coarsely loop divisible for every $\epsilon$ ?

Remark 2.11 describes examples of metric spaces which are not asymptotic cones where the answer to both of these question is no. There are no known examples of finitely generated groups which are coarsely loop divisible but not uniformly coarsely loop divisible which leaves the following question open.

Question 3 Are the conditions uniformly coarsely loop divisible and coarsely loop divisible equivalent for finitely generated groups?

A positive answer to Question 3 would imply a positive answer to Question 1 and show that for finitely generated groups the properties locally simply connected and semilocally simply connected are equivalent.

The property of coarse loop divisibility also allows us to understand general algebraic properties of the fundamental group of an asymptotic cone.

Theorem B (Theorems 2.15, 2.17, Proposition 2.20) If a finitely generated group $G$ is not $\epsilon$-coarsely divisible with respect to $(\omega, d)$ for any $\epsilon>0$ then the fundamental group of $\operatorname{Con}^{\omega}(G, d)$ is uncountable, not free and not simple.

The analogous result holds for hold for all complete homogenous geodesic metric spaces. In Section 2.1 we give a necessary condition to guarantee every asymptotic cone of a given complete homogenous geodesic metric space will satisfy the hypothesis of Theorem B. It turns out that many important groups in the literature satisfy this condition which gives the following corollary.

Corollary C (Corollary 3.2) Let $G$ be a group from the following list. Then for every ultrafilter $\omega$ and scaling sequence $d$; $\operatorname{Con}^{\omega}(G, d)$ is not semilocally simply connected and has an uncountable fundamental group which is not free and not simple. In addition, any decomposition of the fundamental group of $\operatorname{Con}^{\omega}(G, d)$ as a free product has a factor which is not free and uncountable. 
(1) $\mathrm{SL}_{3}(\mathbb{Z})$

(2) Baumslag-Solitar groups: $B S_{p q}=\left\langle a, t \mid t^{-1} a^{p} t=a^{q}\right\rangle$ for $p, q \neq 0$ and $|p| \neq|q|$

(3) The 3-manifold $\mathrm{Sol}_{3}: \mathbb{R}^{3}$ endowed with the Riemannian metric $d s^{2}=e^{2 z} d x^{2}+$ $e^{-2 z} d y^{2}+d z^{2}$

(4) Baumslag-Gertsen group: $\left\langle a, t \mid\left(t^{-1} a^{-1} t\right) a\left(t^{-1} a t\right)=a^{2}\right\rangle$

(5) $\operatorname{Out}\left(F_{n}\right)$ and $\operatorname{Aut}\left(F_{n}\right)$ for $n \geq 3$

(6) $\left\langle a, s, t \mid\left[a, a^{t}\right]=[s, t]=1, a a^{t}=a^{s}\right\rangle$

(7) $\left\langle\theta_{1}, \theta_{2}, a, k \mid a^{\theta_{i}}=a, k^{\theta_{i}}=k a, i=1,2\right\rangle$

The group (6) is of interest since it is metabelian and not polycyclic. It is sometimes referred to as the Baumslag group. The group (7) was constructed by Olshanskii and Sapir and has cubic Dehn function and linear isodiametric function.

\subsection{Definitions}

Let $G=\langle S\rangle$ be a group and $u, v$ be two words in the alphabet $S$. We write $u \equiv v$ when $u$ and $v$ coincide letter by letter and $u={ }_{G} v$ if $u$ and $v$ are equal in $G$. We will denote the Cayley graph of $G$ with respect to the generating set $S$ by $\Gamma(G, S)$. We will use Lab to represent the function from the set of edge paths in a labeled oriented $\mathrm{CW}$ complex to the set of words in the alphabet obtained by reading the label of a path.

Isoperimetric functions Suppose that $\langle S \mid R\rangle$ is a finite presentation for a group $G$. Let $\operatorname{Area}(\Delta)$ denote the number of $R$-cells in a van Kampen diagram $\Delta$. If $w$ is a word in $S \cup S^{-1}$, then $\operatorname{Area}(w)=\min \{\operatorname{Area}(\Delta) \mid \mathbf{L a b}(\partial \Delta) \equiv w\}$. If $\gamma$ is a loop in $\Gamma(G, S)$, then $\operatorname{Area}(\gamma)=\operatorname{Area}(\operatorname{Lab}(\gamma))$.

An isoperimetric function for the presentation $\langle S \mid R\rangle$ of $G$ is a nondecreasing function $\delta: \mathbb{N} \rightarrow[0, \infty)$ such that $\delta(|\partial \Delta|) \geq \operatorname{Area}(\operatorname{Lab}(\partial \Delta))$ for all van Kampen diagrams $\Delta$ over $\langle S \mid R\rangle$. A minimal isoperimetric function of a group is called a Dehn function for $G$.

Two nondecreasing functions $f, g: \mathbb{N} \rightarrow[0, \infty)$ are equivalent, if there exists constants $B, C>0$ such that $f(n) \leq B g(B n+B)+B n+B \leq C f(C n+C)+C n+C$. Up to this equivalence, the Dehn function of a finitely presented group is independent of the finite presentation.

Definition 1.1 (Asymptotic cones) Let $\omega$ be an ultrafilter on $\mathbb{N}$ and $c_{n}$ be a sequence of positive real numbers. The sequence $c_{n}$ is bounded $\omega$-almost surely or $\omega$-bounded, if there exists a number $M$ such that $\omega\left(\left\{n \mid c_{n}<M\right\}\right)=1$. If $c_{n}$ is 
$\omega$-bounded, then there exists a unique number, which we will denote by $\lim ^{\omega} c_{n}$, such that $\omega\left(\left\{n|| c_{n}-\lim ^{\omega} c_{n} \mid<\epsilon\right\}\right)=1$ for every $\epsilon>0$.

If $c_{n}$ is not $\omega$-bounded then $\omega\left(\left\{n \mid c_{n}>M\right\}\right)=1$ for every $M$, in which case we will say that $c_{n}$ diverges $\omega$-almost surely or is $\omega$-divergent and let $\lim ^{\omega} c_{n}=\infty$.

Let $(X$, dist) be a metric space. Let $\omega$ be an ultrafilter on $\mathbb{N}$. Fix an $\omega$-divergent sequence of numbers $d=\left(d_{n}\right)$ called a scaling sequence and a sequence of points $e=\left(e_{n}\right)$ in $X$ called an observation sequence.

Given two sequences $x=\left(x_{n}\right), y=\left(y_{n}\right)$ in $X$, define $\operatorname{dist}(x, y)=\lim ^{\omega} \operatorname{dist}\left(x_{n}, y_{n}\right) / d_{n}$. We can then define an equivalence relation $\sim$ on the set of sequence in $X$ by $x \sim y$ if and only if $\operatorname{dist}(x, y)=0$.

The asymptotic cone of $X$ with respect to $e, d$ and $\omega$ is

$$
\operatorname{Con}^{\omega}(X, e, d)=\left\{x=\left(x_{n}\right) \mid \operatorname{dist}(x, e)<\infty\right\} / \sim .
$$

$\operatorname{Con}^{\omega}(X, e, d)$ is a complete metric space. If $X$ is geodesic then $\operatorname{Con}^{\omega}(X, e, d)$ is also geodesic.

If $X_{n}$ is a sequence of subspaces of $X$ we will use $\lim ^{\omega} X_{n}$ to denote the subspace of $\operatorname{Con}^{\omega}(X, e, d)$ consisting of sequences with representatives in $\prod X_{n}$.

The following observation is well known.

Lemma 1.2 Let $\omega$ be an ultrafilter on $\mathbb{N}, d=\left(d_{n}\right)$ be an $\omega$-divergent sequence of numbers and $e=\left(e_{n}\right)$ an observation sequence. Suppose that $\left\{\gamma_{n}\right\}$ is a sequence of paths parameterized by arc length in a geodesic metric space ( $X$, dist) such that $\left|\gamma_{n}\right|=O\left(d_{n}\right)$ and $\operatorname{dist}\left(e_{n}, \operatorname{im}\left(\gamma_{n}\right)\right) \leq L d_{n}$, for some $L$. Then $\gamma(t)=\left(\gamma_{n}(t)\right)$ is a continuous map into $\operatorname{Con}^{\omega}(X, e, d)$.

The following converse holds and is proved by the second author in [18].

Lemma 1.3 Suppose that $\gamma$ is a path in $\operatorname{Con}^{\omega}(X, e, d)$ where $X$ is a geodesic metric space. Then there exist paths $\gamma_{n}$ in $X$ such that $\gamma(t)=\left(\gamma_{n}(t)\right)$.

Lemma 1.3 does not imply that all geodesics are limits of geodesics since no metric conditions are placed on the limiting functions. In the current article the nomenclature "ball" or "metric" ball will refer to an open ball. We will use $|\tau|$ to denote the arc length of a path. In this setting $|\cdot|$ maps the set of paths into the extended real line, is finite for rectifiable paths and $+\infty$ for nonrectifiable paths. We will assume that rectifiable paths are parameterized proportional to arc length.

The following definitions of locally connectivity properties are standard; see Hatcher [15, Chapter 1]. 
Definition 1.4 A locally path connected space $X$ is called locally simply connected if for every pair $(U, x)$ where $U$ is a neighborhood of $x \in X$, there exists $V$, a neighborhood of $x$ contained in $U$, such that the inclusion induced homomorphism from $\pi_{1}(V, x)$ to $\pi_{1}(U, x)$ is trivial; ie every loop in $V$ bounds a disc in $U$. A locally path connected metric space $X$ is uniformly simply connected if for every $\epsilon>0$ there exists a $\delta>0$ such that every loop with diameter at most $\delta$ bounds a disc with diameter at most $\epsilon$.

A locally path connected space $X$ is called semilocally simply connected if every point $x \in X$ has a neighborhood $U$ such that the inclusion induced homomorphism from $\pi_{1}(U, x)$ to $\pi_{1}(X, x)$ is trivial, ie every loop in $U$ bounds a disc in the whole space.

Remark A space that is locally simply connected is semilocally simply connected. The converse is false, since the cone on any space that is not locally simply connected is semilocally simply connected but still not locally simply connected; see $[15$, Section 1.3].

The following definition of a partition is due to Papasoglu [23].

Partitions of the unit disc in the plane Let $\mathbb{D}$ be the unit disk in $\mathbb{R}^{2}$. A partition $P$ of $\mathbb{D}$ is a finite collection of closed discs $D_{1}, \ldots, D_{k}$ in the plane with pairwise disjoint interiors such that $\mathbb{D}=\bigcup_{i} D_{i}$. Since there are only finitely many discs, the union of the boundaries of the discs forms a connected graph. A point $p$ on $\partial D_{1} \cup \cdots \cup \partial D_{k}$ is called a vertex of the partition if for every open set $U$ containing $p, U \cap\left(\partial D_{1} \cup \cdots \cup \partial D_{k}\right)$ is not homeomorphic to an interval. An edge of a partition is a pair of vertices which are joined by a path in $\partial D_{1} \cup \cdots \cup \partial D_{k}$ that intersects the set of vertices only at its endpoints. We will say that such vertices are adjacent. A piece of a partition is a maximal set of vertices of the partition contained in a single disc of the partition. A partition is then a cellular decomposition of the unit disc where each vertex has degree at least 3; so we will use the standard notation, $P^{(i)}$, to denote the $i^{\text {th }}$ skeleton of a partition for $i=0,1,2$.

Geodesic $n$-gons in a metric space $\mathbf{X}$ An $n$-gon in $X$ is a map from the set of vertices of the standard regular $n$-gon in the plane into $X$, ie an ordered set of $n$ points in $X$. If $X$ is a geodesic metric space, we can extend an $n$-gon to edges by mapping the edge between adjacent vertices of the standard regular $n$-gon in the plane to a geodesics segment joining the corresponding vertices of the $n$-gon in $X$. We will say that such an extension is a geodesic $n-$ gon in $X$.

Partitions of loops in a geodesic metric space $\mathbf{X}$ Let $\gamma: \partial \mathbb{D} \rightarrow X$ be a continuous map. A partition of $\gamma$ is a map $\Pi$ from the set of vertices of a partition $P$ of $\mathbb{D}$ to $X$ 
such that $\left.\Pi\right|_{\partial \mathbb{D} \cap P^{(0)}}=\left.\gamma\right|_{\partial \mathbb{D} \cap P^{(0)}}$. The vertices/edges/pieces of $\Pi$ are the images of vertices/edges/pieces of $P$. We will write $\Pi\left(\partial D_{i}\right)$ for the pieces of $\Pi$, where $D_{i}$ are the 2-cells of $P$.

Remark 1.5 Suppose that $\Pi: P^{(0)} \rightarrow X$ is a partition of a loop $\gamma$ in a geodesic metric space. We can extend $\Pi$ to $P^{(1)}$ by mapping each edge contained in $\partial \mathbb{D}$ to the corresponding subpath of $\gamma$ and every edge not contained in $\partial \mathbb{D}$ to a geodesic segment joining its end points. The length of a piece is the arc length of the loop $\Pi\left(\partial D_{i}\right)$. We will write $\left|\Pi\left(\partial D_{i}\right)\right|$ for the length of the piece $\Pi\left(\partial D_{i}\right)$. We define the mesh of $\Pi$ by

$$
\operatorname{mesh}(\Pi)=\max _{1 \leq i \leq k}\left\{\left|\Pi\left(\partial D_{i}\right)\right|\right\}
$$

When $X$ is a Cayley graph of a group, we will also assume that the partition takes vertices of $P$ to vertices in the Cayley graph. A partition $\Pi$ is called a $\delta$-partition, if mesh $\Pi<\delta$. A loop of length $k$ in a geodesic metric space is partitionable if it has a $\frac{k}{2}$-partition.

Let $P(\gamma, \delta)$ be the minimal number of pieces in a $\delta$-partition of $\gamma$ if a $\delta$-partition exist and $+\infty$ otherwise.

\section{Coarse loop division property}

Definition 2.1 Let $X$ be a geodesic metric space.

Define $\vartheta^{i}: \mathbb{N} \rightarrow \mathbb{N} \cup\{\infty\}$ by $\vartheta^{i}(n)=\sup \left\{P\left(\alpha,|\alpha| / 2^{i}\right) \mid \alpha\right.$ is a loop in $X$ such that $n-1<|\alpha| \leq n\}$. We will call $\vartheta=\vartheta^{1}$ the divisibility function of $X$.

Suppose $\omega$ is an ultrafilter on $\mathbb{N},\left(d_{n}\right)$ an $\omega$-divergent sequence of positive real numbers and $\epsilon$ a positive real number. We will say that $X$ is $\epsilon$-coarsely loop divisible if for every $\delta \in(0, \epsilon)$ there exists an $A \subset \mathbb{N}$ with $\omega(A)=1$ such that the divisibility function $\vartheta$ restricted to $\bigcup_{n \in A}\left[\delta d_{n}, \epsilon d_{n}\right]$ is bounded by a constant $K=K(\delta, \epsilon)$.

We will say that $X$ is uniformly $\epsilon$-coarsely loop divisible if the constant $K=K(\delta, \epsilon)$ can be chosen independent of $\delta$.

We will say that a group $G$ is (uniformly) $\epsilon$-coarsely loop divisible if the Cayley graph $\Gamma(G, S)$ is (uniformly) $\epsilon$-coarsely divisible.

The property of being $\epsilon$-coarsely loop divisible depends on $(\omega, d)$. When there is a chance of confusion, we will say that $X$ is $\epsilon$-coarsely loop divisible with respect to $(\omega, d)$. 
If $X$ is $\epsilon$-coarsely loop divisible for every $\epsilon$ and the bound $K(\delta, \epsilon)$ can be chosen independent of both $\delta$ and $\epsilon$, then $\operatorname{Con}^{\omega}(X, e, d)$ has Olshanskii-Sapir's property $\operatorname{LDC}(K)$ as defined in [21].

We will see (Proposition 2.21) that for finitely generated groups this definition is independent of the generating set in the sense that if $S, S^{\prime}$ are two finite generating sets for $G$, then $\Gamma(G, S)$ is $\epsilon$-coarsely loop divisible if and only if $\Gamma\left(G, S^{\prime}\right)$ is $\epsilon^{\prime}$-coarsely loop divisible for some $\epsilon^{\prime}>0$.

Remark 2.2 Suppose that $\vartheta$ is bounded on $\left[n / 2^{l}, n\right]$ by $K$. Let $\alpha$ be a loop of length $n$ and fix a partition of $\alpha$ into at most $\vartheta(n)$ pieces with mesh less than $\frac{n}{2}$. As in Remark 1.5, the partition can be extended to the 1-skeleton of the partition such that each loop has length less than $\frac{n}{2}$. We can then partition each piece with length at least $\frac{n}{4}$ into at most $K$ pieces of length less than $\frac{n}{4}$. This builds an $\frac{n}{4}$-partition of $\alpha$ with at most $K^{2}$ pieces. Hence $\vartheta^{2}(n) \leq K^{2}$. Iterating this process, we obtain $\vartheta^{l}(n) \leq K^{l}$.

Lemma 2.3 Fix $l \in \mathbb{N}$. If $X$ is $\epsilon$-coarsely loop divisible, then for every $\delta \in(0, \epsilon)$ there exists an $A \subset \mathbb{N}$ with $\omega(A)=1$ such that $\vartheta^{l}$ restricted to $\bigcup_{n \in A}\left[\delta d_{n}, \epsilon d_{n}\right]$ is bounded by a constant $K=K(\delta, \epsilon, l)$.

Thus the coarse loop division property does not depend on which function $\vartheta^{l}$ is used in its definition.

Proof Suppose $X$ is $\epsilon$-coarsely loop divisible. Fix $\delta$ such that $0<\delta<\epsilon$. Choose a $K$ and an $\omega$-large $A$ such that $\vartheta$ restricted to $\bigcup_{n \in A}\left[\left(\delta / 2^{l}\right) d_{n}, \epsilon d_{n}\right]$ is bounded by $K$. By Remark 2.2, $\vartheta^{l}$ restricted to $\bigcup_{n \in A}\left[\delta d_{n}, \epsilon d_{n}\right]$ is bounded by $K^{l}$.

Definition 2.4 Let $\left(\gamma_{n}\right)$ be a sequence of loops in a metric space $X$ and $d=\left(d_{n}\right)$ an $\omega$-divergent sequence of real numbers. Then $\left(\gamma_{n}\right)$ is not $(m, d, \epsilon, \delta)$-partitionable if $\delta d_{n} \leq\left|\gamma_{n}\right| \leq \epsilon d_{n}$ and $P\left(\gamma_{n},\left|\gamma_{n}\right| / 2\right)>m \omega$-almost surely. When $d$ and $\epsilon$ are fixed, we will say that $\left(\gamma_{n}\right)$ is not $(\delta, m)$-partitionable. Additionally, given a sequence of loops which is not $(\delta, m)$-partitionable, we will say that a fixed member $\gamma_{n}$ of the sequence is not $(\delta, m)$-partitionable if $\delta d_{n} \leq\left|\gamma_{n}\right| \leq \epsilon d_{n}$ and $P\left(\gamma_{n},\left|\gamma_{n}\right| / 2\right)>m$.

Remark 2.5 Let $\gamma: \partial \mathbb{D} \rightarrow X$ be parameterized by arc length. Suppose that we have $8 \operatorname{diam}(\gamma)<|\gamma|$. Let $P$ be the cellular decomposition of the unit disc $\mathbb{D}$ such that $P^{(1)}$ is $\partial \mathbb{D} \cup A$ where $A$ is a maximal square inscribed in $\mathbb{D}$. Then $\Pi: P^{(0)} \rightarrow X$ defined by $\Pi(t)=\gamma(t)$ is a partition of $\gamma$ with five pieces (four 2-gons and one 4-gon) and $\operatorname{mesh}(\Pi) \leq \max \left\{\frac{|\gamma|}{4}+\operatorname{diam}(\gamma), 4 \operatorname{diam}(\gamma)\right\}<\frac{|\gamma|}{2}$.

Thus, if $\left(\gamma_{n}\right)$ is not $(m, d, \epsilon, \delta)$-partitionable for some $m \geq 5$, then $\left|\gamma_{n}\right| \leq 8 \operatorname{diam}\left(\gamma_{n}\right)$. Hence if $\left(\gamma_{n}\right)$ is not $(\delta, m)$-partitionable, then $\left|\gamma_{n}\right| \leq O\left(\operatorname{diam}\left(\gamma_{n}\right)\right)$ where the big $O$ constant is independent of $\left(\gamma_{n}\right)$. 
The following two propositions were proved by Papasoglu in [23, pages 792-793]. The formulations are slightly different here but the proofs are the same. The proofs are also outlined in [21].

Proposition 2.6 Let $X$ be a metric space and $\left(\gamma_{n}\right)$ a sequence of loops in $X$ such that $\left|\gamma_{n}\right|=O\left(d_{n}\right)$ and $\operatorname{dist}\left(e_{n}, \operatorname{im}\left(\gamma_{n}\right)\right) \leq L d_{n}$, for some $L$. If each $\gamma_{n}$ has a $\delta_{n}$-partition with at most $k$ pieces, then the loop $\gamma(t)=\left(\gamma_{n}(t)\right)$ in $\operatorname{Con}^{\omega}(X, e, d)$ has a $\delta$-partition with at most $k$ pieces where $\delta=\lim ^{\omega}\left(\delta_{n} / d_{n}\right)$.

Proposition 2.7 Let $X$ be a complete geodesic metric space. If $X$ is uniformly $\epsilon-$ coarsely loop divisible for every $\epsilon>0$ with respect to the pair $(\omega, d)$, then $\operatorname{Con}^{\omega}(X, e, d)$ is simply connected for any observation sequence $e$.

To prove Proposition 2.7, Papasoglu uses Proposition 2.6 to show that every loop in $\operatorname{Con}^{\omega}(X, e, d)$ is partitionable and the number of pieces is independent of the loop. He then iterates the process of taking partitions and extending them to the 1 -skeleton as in Remark 1.5. A consequence of this procedure is that the diameter of the constructed disc is proportional to the length of the loop (the proportionality constant can be chosen to be the bound on the number of pieces in the partitions).

Lemma 2.8 Suppose that $X$ is a complete geodesic metric space which is uniformly $\epsilon$-coarsely loop divisible with respect to the pair $(\omega, d)$. Then there exists a constant $K$ such that, for any observation sequence $e$, every loop in $\operatorname{Con}^{\omega}(X, e, d)$ with diameter less than $\frac{\epsilon}{8}$ bounds a disc with diameter less than $K \epsilon$.

Proof Since $X$ is uniformly $\epsilon$-coarsely loop divisible every loop in $\operatorname{Con}^{\omega}(X, e, d)$ with length less than $\epsilon$ is partitionable with a uniform bound on the number of pieces required. Suppose that a loop in $\operatorname{Con}^{\omega}(X, e, d)$ has length at least $\epsilon$ and diameter less than $\frac{\epsilon}{8}$. Then it has a partition with 5 pieces by Remark 2.5. Thus every loop in $\operatorname{Con}^{\omega}(X, e, d)$ with diameter less than $\frac{\epsilon}{8}$ is partitionable and we can apply the proof of Proposition 2.7.

Lemma 2.8 can be restated in the following way.

Proposition 2.9 Let $X$ be a complete geodesic metric space. If $X$ is uniformly $\epsilon-$ coarsely loop divisible, then $\operatorname{Con}^{\omega}(X, e, d)$ is uniformly locally simply connected for any observation sequence $e$.

Proposition 2.10 Let $X$ be a complete geodesic metric space. If $X$ is uniformly $\epsilon$-coarsely loop divisible, then $X$ has an asymptotic cone which is simply connected. 
Proof Suppose that $X$ is uniformly $\epsilon$-coarsely loop divisible for some $(\omega, d)$ and $\epsilon>0$. We can consider an ultralimit of the metric spaces $X_{k}=\operatorname{Con}^{\omega}\left(X, e,\left(d_{n} / k\right)\right)$. By Druțu and Sapir [8, Corollary 3.24], $\lim ^{\omega} X_{k}$ is again an asymptotic cone of $X$. Thus we can choose $\left(\mu,\left(p_{n}\right)\right)$ and $\left(x_{n}\right)$ such that $\operatorname{Con}^{\mu}\left(X,\left(x_{n}\right),\left(p_{n}\right)\right)$ and $\lim ^{\omega} X_{k}$ are isometric.

The identity map id from $\operatorname{Con}^{\omega}(X, e, d)$ to $X_{k}$ rescales distances by a fixed constant which implies that $P(\gamma,|\gamma| / 2)=P(\operatorname{id}(\gamma), \operatorname{id}(|\gamma|) / 2)$ for every $\gamma \in \operatorname{Con}^{\omega}(X, e, d)$. Since $X$ is uniformly $\epsilon$-coarsely loop divisible, there is a $v_{0}$ such that $P(\gamma,|\gamma| / 2)<v_{0}$ for every loop $\gamma$ contained in a ball of radius $\frac{\epsilon}{4}$ in $\operatorname{Con}^{\omega}(X, e, d)$. Hence every loop $\gamma$ contained in a ball of radius $\frac{k \epsilon}{4}$ in $X_{k}$ has the property that $P(\gamma,|\gamma| / 2)<v_{0}$. Thus for any loop $\alpha$ in $\lim ^{\omega} X_{k} ; P(\alpha,|\alpha| / 2)<v_{0}$. Hence $\lim ^{\omega} X_{k}$ is uniformly $\epsilon$-coarsely loop divisible for every $\epsilon>0$ with respect to the pair $\left(\mu,\left(p_{n}\right)\right)$ and Proposition 2.7 implies that $\operatorname{Con}^{\mu}\left(X,\left(x_{n}\right),\left(p_{n}\right)\right)$ is simply connected.

Lemma 2.8 shows that a necessary condition for a group to be uniformly $\epsilon$-coarsely loop divisible for every $\epsilon>0$ is that all loops in $\operatorname{Con}^{\omega}(X, e, d)$ bound discs with diameters proportional to their length.

Remark 2.11 Let $X$ be a topological space. The topological cone of $X$ written $\hat{X}$ is the quotient space of $X \times[0,1]$ obtained by identifying all points $(x, 1)$ for $x \in X . X$ canonically embeds in $\hat{X}$ by $x \mapsto(x, 0)$ and we will generally identify $X$ with $X \times\{0\}$. The Hawaiian earring is the one-point compactification of a sequence of disjoint arcs and can be realized in the plane as the union of circles centered at $\left(0, \frac{1}{n}\right)$ with radius $\frac{1}{n}$. We will use $\boldsymbol{E}$ to denote this subspace of the plane and $\boldsymbol{a}_{n}$ to denote the circle centered at $\left(0, \frac{1}{n}\right)$ with radius $\frac{1}{n}$. The Hawaiian earring group is $\pi_{1}(\boldsymbol{E},(0,0))=\mathbb{H}$. Let $\boldsymbol{E}_{n}=\bigcup_{i \geq n} \boldsymbol{a}_{i}$ and $\mathbb{H}_{n}=\pi_{1}\left(\boldsymbol{E}_{n},(0,0)\right) \leq \mathbb{H}$. Notice that $\boldsymbol{E}_{n}$ is homeomorphic to $\boldsymbol{E}$ which implies that $\mathbb{H}_{n}$ is isomorphic to $\mathbb{H}$.

The space $\hat{\boldsymbol{E}}$ is not uniformly $\epsilon$-coarsely loop divisible but is simply connected and not locally simply connected. Suppose that instead of coning from a single point, we were to cone each circle individually. Then as long as we required that the sequence of cone points converged to the wedge point of $\boldsymbol{E}$ but at a rate slower than the radii of the loops, this space would be locally simply connected but not be uniformly $\epsilon$-coarsely loop divisible for any $\epsilon$. These two examples show that for general metric spaces being uniformly $\epsilon$-coarsely loop divisible is not a necessary condition for a space to be simply connected or locally simply connected.

Erschler and Osin [11] and Druțu and Sapir [8] proved that many metric spaces $\pi_{1}-$ embed into the asymptotic cones of finitely generated groups. In both papers, the spaces 
that were $\pi_{1}$-embedded into the asymptotic cones of finitely generated groups were uniformly locally simply connected.

A positive answer to either Question 1 or 2 would imply that the results of Erschler-Osin and Druțu-Sapir cannot be extended to spaces which are semilocally simply connected but not locally simply connected.

We will now prove some implications of the coarse loop division property.

The following lemma is an immediate consequence of Proposition 2.6 and Lemma 1.3.

Lemma 2.12 Suppose that $X$ is a complete geodesic metric space which is $\epsilon-$ coarsely loop divisible. Then, for any choice of observation sequence $e$, every loop in $\operatorname{Con}^{\omega}(X, e, d)$ with length less than $\epsilon$ is partitionable.

Lemma 2.13 Suppose that $X$ is a complete homogeneous geodesic metric space. If every loop in $\operatorname{Con}^{\omega}(X, e, d)$ with length less than $\epsilon$ is partitionable, then $X$ is $\epsilon^{\prime}$-coarsely loop divisible with respect to the pair $(\omega, d)$ for every $\epsilon^{\prime}<\epsilon$.

Proof Suppose that $X$ is not $\epsilon^{\prime}$-coarsely loop divisible with respect to the pair $(\omega, d)$ for some $\epsilon^{\prime}$ with $0<\epsilon^{\prime}<\epsilon$. Then there exists a $\delta>0$ such that for every $\omega$-large $A, \vartheta$ restricted to $\bigcup_{n \in A}\left[\delta d_{n}, \epsilon^{\prime} d_{n}\right]$ is unbounded.

Let $\gamma_{n}$ be a loop based at $e_{n}$ such that $\delta d_{n} \leq\left|\gamma_{n}\right| \leq \epsilon^{\prime} d_{n}$ and satisfies at least one of the two following properties.

(a) $P\left(\gamma_{n}, \frac{\left|\gamma_{n}\right|}{2}\right)>n$

(b) $P\left(\gamma_{n}, \frac{\left|\gamma_{n}\right|}{2}\right) \geq P\left(\alpha, \frac{|\alpha|}{2}\right)$ for all $\alpha$ such that $\delta d_{n} \leq|\alpha| \leq \epsilon^{\prime} d_{n}$

Let $m_{n}=P\left(\gamma_{n},\left|\gamma_{n}\right| / 2\right)$. Since $\vartheta$ restricted to $\bigcup_{n \in A}\left[\delta d_{n}, \epsilon d_{n}\right]$ is unbounded for every $\omega$-large $A, \lim ^{\omega} m_{n}=+\infty$. Thus for every $m,\left(\gamma_{n}\right)$ is not $(\delta, m)$-partitionable.

The path $\gamma(t)=\left(\gamma_{n}(t)\right)$ is a well-defined loop in $\operatorname{Con}^{\omega}(X, e, d)$ with positive diameter and arc length at most $\epsilon^{\prime}<\epsilon$. By assumption, there is a $\frac{|\gamma|}{2}$-partition of $\gamma$ with $L$ pieces. However, this induces a $\left|\gamma_{n}\right| / 2$-partition of $\gamma_{n}$ with $L$ pieces $\omega$-almost surely. Hence $P\left(\gamma_{n},\left|\gamma_{n}\right| / 2\right) \leq L \omega$-almost surely, which contradicts our choice of $m_{n}$.

Proposition 2.14 Suppose that $X$ is a complete homogenous geodesic metric space. If $\operatorname{Con}^{\omega}(X, e, d)$ is semilocally simply connected then $X$ is $\epsilon$-coarsely loop divisible for some $\epsilon>0$.

It is not known whether the converse holds. The converse is Question 2 with the uniform hypothesis removed. 
Proof of Proposition 2.14 Suppose every loop in $\operatorname{Con}^{\omega}(X, e, d)$ contained in a ball of radius $\epsilon$ is nullhomotopic in $\operatorname{Con}^{\omega}(X, e, d)$. Then for every $\gamma$ of length at most $\epsilon$, there exists a continuous map of a disc into $\operatorname{Con}^{\omega}(X, e, d)$ which extends $\gamma$ and is necessarily uniformly continuous. For sufficiently small $v$, a $v$-partition of the disc gives us a finite $|\gamma| / 2$-partition for $\gamma$. Then the result follows from Lemma 2.13

Theorem 2.15 Let $X$ be a complete homogenous geodesic metric space. If $X$ is not $\epsilon$-coarsely loop divisible with respect to $(\omega, d)$ for every $\epsilon>0$, then $\operatorname{Con}^{\omega}(X, e, d)$ has uncountable fundamental group.

The proof will require the following result of Cannon and Conner.

Theorem 2.16 [4] Let $X$ be a topological space, $\varphi: \pi_{1}\left(X, x_{0}\right) \rightarrow L$ be a homomorphism to a group $L, U_{1} \supset U_{2} \supset \cdots$ be a countable local basis for $X$ at $x_{0}$ and $G_{i}$ be the image of the natural map from $\pi_{1}\left(U_{i}, x_{0}\right)$ into $\pi_{1}\left(X, x_{0}\right)$. If $L$ is countable, then the sequence $\varphi\left(G_{1}\right) \supset \varphi\left(G_{2}\right) \supset \cdots$ is eventually constant.

Proof of Theorem 2.15 Let $X$ be a complete homogenous geodesic metric space. Suppose that $X$ is not $\epsilon$-coarsely loop divisible for any $\epsilon$ and $\operatorname{Con}^{\omega}(X, e, d)=X^{\omega}$ has countable fundamental group. Let $i_{*}$ be the identity map on $\pi_{1}\left(X^{\omega}, e\right)$. Theorem 2.16 implies that $i_{*}\left(G_{n}\right)$ is eventually constant, where $G_{n}$ is the image of the natural map from $\pi_{1}\left(B_{1 / n}(e), e\right)$ into $\pi_{1}\left(X^{\omega}, e\right)$.

Fix $N$ such that this sequence is constant for $m \geq N$, and let $\epsilon=1 / N$. Therefore every loop in $B_{1 / N}(\tilde{x})$ can be homotoped into $B_{1 / m}(e)$ for any $m \geq N$. In general, this will not imply that the ball is simply connected. However, it does imply that every loop $\gamma$ of length less than $\epsilon$ has a partition with finitely many pieces and mesh at most $\frac{|\gamma|}{2}$. Then Lemma 2.13 implies that $X$ is $\epsilon$-coarsely loop divisible which is a contradiction.

Theorem 2.17 Let $X$ be a complete homogenous geodesic metric space. Fix a ultrafilter $\omega$ and scaling sequence $d$. If, for every $\epsilon>0, X$ is not $\epsilon$-coarsely loop divisible with respect to the pair $(\omega, d)$, then the fundamental group of $\operatorname{Con}^{\omega}(X, e, d)$ is not free. Evenmore, if $\pi_{1}\left(\operatorname{Con}^{\omega}(X, e, d)\right)=*_{j} G_{j}$ for some free product of groups $G_{j}$, then there exists a $j$ such that $G_{j}$ is uncountable and not free.

We will use the following two results in the proof of Theorem 2.17.

Theorem 2.18 Suppose that $\varphi: \mathbb{H} \rightarrow \mathbb{F}$ is a surjective homomorphism where $\mathbb{F}$ is a free group. Then $\mathbb{F}$ has finite rank. 
If we consider homomorphisms from the natural inverse limit containing $\mathbb{H}$ to free groups, then this is a theorem of Higman [16]. When we consider homomorphism from $\mathbb{H}$, this is a consequence of Theorem 2.16 and a proof can be found in Summers [27].

Theorem 2.19 (Eda [9]) Suppose that $\varphi: \mathbb{H} \rightarrow *_{j} G_{j}$ is a homomorphism. Then there exists an $n$ such that $\varphi\left(\mathbb{H}_{n}\right)$ is contained in a subgroup which is conjugate to $G_{j}$ for some $j$.

Proof of Theorem 2.17 Since $X$ is not $\epsilon$-coarsely loop divisible with respect to $(\omega, d)$ for every $\epsilon$, we may find a null sequence of loops $\alpha_{n}$ in $\operatorname{Con}^{\omega}(X, e, d)$ such that $\alpha_{n}$ has no finite $\left(\left|\alpha_{n}\right| / 2\right)$-partition. Since $\operatorname{Con}^{\omega}(X, e, d)$ is transitive by isometries, we may choose $\alpha_{n}$ such that $\alpha_{i}(0)=\alpha_{j}(0)=e$ for all $i, j$. By passing to a subsequence, we may assume that $\left|\alpha_{n}\right|<\left|\alpha_{n-1}\right| / 2$. This implies that the ball of radius $\left|\alpha_{n}\right|$ does not contain a loop which is homotopic to $\alpha_{i}$ for $i<n$. Since $\alpha_{n}$ forms a null sequence of loops and $\alpha_{i}(0)=\alpha_{j}(0)$ for all $i, j$, there exists a continuous map $f$ from $\boldsymbol{E}$ to $\operatorname{Con}^{\omega}(X, e, d)$ such that $f\left(\boldsymbol{a}_{n}\right)=\alpha_{n}$.

Suppose that $\pi_{1}\left(\operatorname{Con}^{\omega}(X, e, d),\left(x_{n}\right)\right)$ was free. Then $f_{*}(\mathbb{H})$ would be free and Theorem 2.18 would then imply that it has finite rank. Hence $f_{*}(\mathbb{H})$ is countable and Theorem 2.16 implies that $f_{*}\left(\pi_{1}\left(\boldsymbol{E}_{n},(0,0)\right)\right)$ as a sequence in $n$ is eventually constant which contradicts our choice of $\alpha_{n}$.

Thus for every $n, f_{*}\left(\mathbb{H}_{n}\right)$ is uncountable and not free. The last claim of the theorem follows from Theorem 2.19.

Proposition 2.20 Let $X$ be a complete homogenous geodesic metric space. If $X$ is not $\epsilon$-coarsely loop divisible with respect to $(\omega, d)$ for every $\epsilon>0$, then the fundamental group of $\operatorname{Con}^{\omega}(X, e, d)$ is not simple.

Proof Let $X^{\omega}=\operatorname{Con}^{\omega}(X, e, d)$ and $\alpha_{i}$ be a null sequence of loops in $X^{\omega}$ constructed as in the proof of Theorem 2.17. Let $A_{n}$ be the union of the images of $\alpha_{i}$ for $i>n$. Let $Y_{n}$ be the topological cone of $A_{n}$ in $X^{\omega}$, ie the subset of $\hat{X}^{\omega}$ consisting of $\operatorname{Con}^{\omega}(X, e, d) \times\{0\}$ and the canonically embedded $\hat{A}_{n}$. The inclusion map $\iota_{n}: \operatorname{Con}^{\omega}(X, e, d) \rightarrow Y_{i}$ defined by $x \mapsto(x, 0)$ induces a map $\iota_{n *}$ on fundamental groups with nontrivial kernel. Hence, it is enough to show that the induced map on fundamental groups is nontrivial.

Claim For $i \leq n, \iota_{n}\left(\alpha_{i}\right)$ is homotopically essential in $Y_{n}$. 
Proof of claim Suppose that $h: \mathbb{D} \rightarrow Y_{n}$ is a nullhomotopy of $\iota_{n}\left(\alpha_{i}\right)$ for some $i \leq n$ where $\mathbb{D}$ is the unit disk in the plane. Let $z$ be the cone point. Notice that $A_{n}$ separates $Y_{n}$. Hence the boundary of each component of $h^{-1}\left(\hat{A}_{n}\right)$ is contained in $h^{-1}\left(A_{n}\right)$. By possibly modifying $h$, we may assume that each component of $h^{-1}\left(\hat{A}_{n}\right)$ which is not contained in $h^{-1}\left(A_{n}\right)$ intersects the cone point $z$. (Suppose $B$ is a component of $h^{-1}\left(\hat{A}_{n}\right)$ such that $h(B) \cap\{z\}=\varnothing$. Then we can push $h$ down along cone lines to insure that $h(B) \subset A_{n}$.)

Since each component of $h^{-1}\left(\hat{A}_{n}\right)$ which is not contained in $h^{-1}\left(A_{n}\right)$ intersects $h^{-1}\left(A_{n}\right)$ and $h^{-1}(z)$ (two disjoint closed sets), there are only finitely many components of $h^{-1}\left(\widehat{A}_{n}\right)$ which are not contained in $h^{-1}\left(A_{n}\right)$.

Let $C$ be the component of $h^{-1}\left(X^{\omega}\right)$ containing the unit circle in the plane. Then $C$ is a planar annulus of finite genus. (The genus is equal to the number of components of $h^{-1}\left(\hat{A}_{n}\right)$ which are not contained in $h^{-1}\left(A_{n}\right)$.) Since each boundary component of $C$ except the unit circle maps into $A_{n}$, the diameter of its image is at most $\left|\alpha_{n+1}\right|<\left|\alpha_{n}\right| / 2$. This implies that $h: C \rightarrow X^{\omega}$ can be used to find a finite partition of $\alpha_{i}$ with mesh at most $\left|\alpha_{n}\right| / 2$. Hence, $\alpha_{i}$ is partitionable which contradicts our choice of $\alpha_{i}$.

The property of being $\epsilon$-coarsely loop divisible is a quasi-isometry invariant in the following sense.

Proposition 2.21 If $X$ and $Y$ are two quasi-isometric homogenous geodesic metric spaces, then $X$ is $\epsilon$-coarsely loop divisible if and only if $Y$ is $\epsilon^{\prime}$-coarsely loop divisible for some $\epsilon^{\prime}>0$.

Proof If $X$ and $Y$ are quasi-isometric, then their cones are bilipschitz. If $X$ is $\epsilon$-coarsely loop divisible for some $\epsilon>0$, then Proposition 2.6 implies that every loop of length less than $\epsilon$ in $\operatorname{Con}^{\omega}(X, e, d)$ is partitionable.

Let $f: \operatorname{Con}^{\omega}(X, e, d) \rightarrow \operatorname{Con}^{\omega}\left(Y, e^{\prime}, d\right)$ be a bilipschitz map with bilipschitz constant $C$. By iterating partitions as in Remark 2.2, we can see that every loop of length less than $\epsilon$ in $\operatorname{Con}^{\omega}(X, e, d)$ has a partition with finitely many pieces and mesh at most $\frac{|\gamma|}{2 C}$. Let $\gamma$ be a loop in $\operatorname{Con}^{\omega}\left(Y, e^{\prime}, d\right)$ with length less than $\frac{\epsilon}{C}$. Then $f^{-1} \circ \gamma$ has length at most $\epsilon$ and hence has a partition with mesh at most $\frac{|\gamma|}{2 C}$. Then composing the partition with $f$ gives us a partition of $\gamma$ with finitely many pieces and mesh at most $\frac{|\gamma|}{2}$. Lemma 2.13 implies that $Y$ is $\epsilon^{\prime}$-coarsely loop divisible for every $\epsilon^{\prime}<\frac{\epsilon}{C}$.

\subsection{Absolutely nondivisible sequences}

Definition 2.22 A sequence of loops $\left(\alpha_{n}\right)$ is absolutely nondivisible if there exists an $M$ such that the sequences $P\left(\alpha_{n},\left|\alpha_{n}\right| / M\right)$ and $\left|\alpha_{n}\right|$ both tend to $+\infty$ and $\left\{\left|\alpha_{n+1}\right| /\left|\alpha_{n}\right|\right\}$ is bounded. 
Remark 2.23 Suppose that $\left|\alpha_{n}\right|$ is unbounded and $\left\{\left|\alpha_{n+1}\right| /\left|\alpha_{n}\right|\right\}$ is bounded. To simplify our notation, we will let $\left|\alpha_{n}\right|=a_{n}$ and $B$ be a bound on $\left\{a_{n+1} / a_{n}\right\}$.

Let $n_{0}=1$. Then we can define $\left\{n_{i}\right\}$, inductively, by $n_{i+1}=\min \left\{n \in \mathbb{N} \mid a_{n}>a_{n_{i}}+1\right.$ and $n>n_{i}$ \}. If $n_{i+1} \neq n_{i}+1$, then $a_{k} \leq a_{n_{i}}+1$ for all $n_{i} \leq k<n_{i+1}$.

Thus

$$
\frac{a_{n_{i+1}}}{a_{n_{i}}}=\frac{a_{n_{i+1}}}{a_{\left(n_{i+1}\right)-1}} \cdot \frac{a_{\left(n_{i+1}\right)-1}}{a_{n_{i}}} \leq B \frac{a_{n_{i}}+1}{a_{n_{i}}} \leq B \max \left\{2, \frac{2}{a_{n_{0}}}\right\} .
$$

Therefore $\left\{a_{n_{i}}\right\}_{i}$ is a subsequence which is absolutely nondivisible.

Thus, it is possible to loosen this definition slightly and only require that $\left|\alpha_{n}\right|$ be unbounded.

Lemma 2.24 Fix $\omega$ an ultrafilter on $\mathbb{N}, d$ an $\omega$-divergent sequence and $A$ an infinite subset of the natural numbers. Suppose that $A=\left\{b_{1}<b_{2}<b_{3}<\cdots\right\}$ has the property that the set of ratios $\left\{b_{k+1} / b_{k}\right\}$ is bounded by $L$. Then for any $\epsilon>0$, there exists a sequence $\left(a_{n}\right)$ in $A$ such that $\lim ^{\omega} a_{n} / d_{n} \in\left[\frac{\epsilon}{L}, \epsilon\right]$.

We allow $a_{n}$ to have repeated terms; hence, $a_{n}$ is not necessarily a subsequence of $b_{n}$. However $a_{n}$ is not eventually constant, since $\lim ^{\omega} d_{n}=+\infty$.

Proof Let $L$ be an upper bound on the set $\left\{b_{n+1} / b_{n}\right\}$. For all $n$ such that $b_{1} / d_{n} \leq \epsilon$, choose $\left(i_{n}\right)$ such that $b_{i_{n}} / d_{n} \leq \epsilon<b_{i_{n}+1} / d_{n}$. Let $a_{n}=b_{i_{n}}$.

Then $\epsilon d_{n}<b_{i_{n}+1}$ which implies that $\epsilon / L<b_{i_{n}} / d_{n}=a_{n} / d_{n} \leq \epsilon$. For all $n$ such that $b_{1} / d_{n}>\epsilon$, let $a_{n}=b_{1}$. Then $\lim ^{\omega} a_{n} / d_{n} \in\left[\frac{\epsilon}{L}, \epsilon\right]$.

Lemma 2.25 Let $X$ be a complete geodesic metric space. If there exists a sequence of absolutely nondivisible loops in $X$, then for every pair $(\omega, d)$ and $\epsilon>0, X$ is not $\epsilon$-coarsely loop divisible.

Proof Fix $\epsilon>0, \omega$ an ultrafilter and $d$ an $\omega$-divergent sequence of real numbers.

Let $\left(\gamma_{n}\right)$ be a sequence of loops in $X$ which is absolutely nondivisible. By passing to a subsequence as in Remark 2.23, we may assume that the lengths of $\gamma_{n}$ are nondecreasing. Let $A=\left\{\left|\gamma_{n}\right|\right\}$ and $L$ be an upper bound on $\left\{\left|\gamma_{n+1}\right| /\left|\gamma_{n}\right|\right\}$.

Let $\left(a_{n}\right) \subset A$ be a sequence constructed as in Lemma 2.24 where we replace $\epsilon$ by $\frac{\epsilon}{2}$. Consider the sequence of loops $\gamma_{k_{n}}$ where $\gamma_{k_{n}}$ has length $a_{n}$. Since $\lim ^{\omega} a_{n} / d_{n} \in$ $\left[\frac{\epsilon}{2 L}, \frac{\epsilon}{2}\right]$, we have $\left|\gamma_{k_{n}}\right| \in\left[d_{n} \epsilon / L, d_{n} \epsilon\right] \omega$-almost surely. However, $P\left(\gamma_{k_{n}},\left|\gamma_{k_{n}}\right| / M\right)$ tends to $+\infty$. Hence, $\vartheta$ restricted to $\bigcup_{n \in A}\left[\epsilon / L d_{n}, \epsilon d_{n}\right]$ is unbounded for all $\omega-$ large $A$. Hence Lemma 2.3 implies that $X$ is not $\epsilon$-coarsely loop divisible. Since $\epsilon$ was arbitrary, $X$ is not $\epsilon$-coarsely loop divisible with respect to $(\omega, d)$ for any $\epsilon>0$. Since $(\omega, d)$ were also arbitrary, this completes the proof. 
Lemma 2.25 and Theorem 2.15 immediately imply the following corollary.

Corollary 2.26 Let $X$ be a complete homogenous geodesic metric space. If there exists a sequence of loops in $X$ which is absolutely nondivisible, then every asymptotic cone of $X$ has uncountable fundamental group and is not semilocally simply connected at any point.

\subsection{Simply connected cones}

When Papasoglu proved Proposition 2.7, he used the uniform bound on the number of pieces in a partition to construct discs. Being coarsely loop divisible implies that loops in the cone are partitionable but does not give a bound on the number of pieces which is independent of the loop. Thus Papasoglu's method is insufficient to build discs when a space is only coarsely loop divisible and not uniformly coarsely loop divisible. Here we will show that requiring a linear isodiametric function on partitions along with coarsely loop divisible is sufficient to build discs.

When considering subsets of $\mathbb{N}$, we will write $[a, b]$ for the set $\{n \in \mathbb{Z} \mid a \leq n \leq b\}$. For $A \subset \mathbb{N}$, we will let $A^{c}=\mathbb{N} \backslash A$. For $d \in \mathbb{R}^{+}$and $A \subset \mathbb{N}$, let $\mathcal{M}_{d}(A)=$ $\left\{x \in N \mid\left[\frac{x}{d}, x d\right] \cap A \neq \varnothing\right\}$.

Proposition 2.27 Suppose that for every $\left(\mu,\left(p_{n}\right)\right)$ there exists an $\epsilon>0$ such that $X$ is $\epsilon$-coarsely loop divisible with respect to the pair $\left(\mu,\left(p_{n}\right)\right)$. Then there exists a pair $(\omega, d)$ such that $X$ is $\epsilon$-coarsely loop divisible for every $\epsilon>0$ with respect to $(\omega, d)$.

Before we can prove Proposition 2.27, we will need a necessary condition for $X$ to be $\epsilon$-coarsely loop divisible for every pair $(\omega, d)$.

Lemma 2.28 Let $A_{k}=\vartheta^{-1}([1, k]), A_{k}^{\prime}=\vartheta^{-1}(\{k\})$ and $B_{k}=\vartheta^{-1}([k+1, \infty))$. If $X$ is $\epsilon$-coarsely loop divisible for every pair $(\omega, d)$, then for every $s \in \mathbb{N}$ there exists $b=b(s)$ such that

(i) if $c_{k}^{i}=\sup \left\{\frac{y}{x} \mid i<x\right.$ and $\left.[x, y] \subset A_{k}\right\}$, then $c_{k}=\lim _{i \rightarrow \infty} c_{k}^{i}$ and $c_{k} \rightarrow \infty$,

(ii) if $b_{k}=\sup \left\{\frac{y}{x} \mid[x, y] \subset \mathcal{M}_{s}\left(B_{k}\right)\right\}$, then $b_{k}<b$ for all sufficiently large $k$,

(iii) if $c_{k}^{\prime}=\left\{\frac{y}{x} \mid[x, y] \subset \mathcal{M}_{s}\left(A_{k}^{\prime}\right)\right\}$, then $c_{k}^{\prime}<\alpha$ for all $k$.

Proof For fixed $k, c_{k}^{i}$ is a decreasing sequence in $i$. Hence, $c_{k}$ exists as an extended real number ( $c_{k}^{i}$ might be infinite for all $i$ ). The sequence $c_{k}$ is increasing since the sets $A_{k}$ are nested. 
Proof of (i) Suppose that there existed $L$ such that $c_{k}<L$ for all $k$. We may choose an increasing sequence $k_{n}$ such that $c_{n}^{i}<2 L$ for all $i>k_{n}$. Thus for every interval $[x, y]$ such that $k_{n}<x$ and $\frac{x}{y} \geq 2 L,[x, y] \not \subset A_{n}$, ie $[x, y] \cap B_{n} \neq \varnothing$.

Fix an ultrafilter $\omega$ and let $d_{n}=\left(k_{n}\right)^{2}$. Suppose $X$ is $\epsilon$-coarsely loop divisible for some $\epsilon>0$. Then $\bigcup_{n \in A}\left[\epsilon d_{n} /(2 L), \epsilon d_{n}\right] \subset A_{t}$ for some $t$ and $\omega$-large $A$. However, for all sufficiently large $n, k_{n}<\epsilon d_{n} /(2 L)$ which implies that $\left[\epsilon d_{n} /(2 L), \epsilon d_{n}\right] \cap$ $B_{n} \neq \varnothing$ for all sufficiently large $n$. This contradicts our choice of $t$ such that $\bigcup_{n \in A}\left[\epsilon d_{n} /(2 L), \epsilon d_{n}\right] \subset A_{t}$.

Proof of (ii) Fix $s \in \mathbb{N}$. Suppose that (ii) does not hold. Then there exists $\left[x_{n}, y_{n}\right] \subset$ $\mathcal{M}_{s}\left(B_{n}\right)$ such that $y_{n} / x_{n}>n$. Fix an ultrafilter $\omega$ and let $d_{n}=\left(x_{n} y_{n}\right)^{1 / 2}$, the geometric center of the interval $\left[x_{n}, y_{n}\right]$. Then for every $n^{\prime} \leq n, \mathcal{M}_{\sqrt{n}}\left(d_{n}\right) \subset\left[x_{n}, y_{n}\right] \subset$ $\mathcal{M}_{S}\left(B_{n^{\prime}}\right)$. (The first inclusion follows by our choice of $d_{n}$ and the second holds since $\mathcal{M}_{S}\left(B_{n}\right) \subset \mathcal{M}_{S}\left(B_{n^{\prime}}\right)$ for $n^{\prime} \leq n$.)

Suppose $X$ is $\epsilon$-coarsely loop divisible with respect to $(\omega, d)$ for some $\epsilon \in(0,1)$. For any $0<\delta<\epsilon, \bigcup_{n>m}\left[\delta d_{n}, \epsilon d_{n}\right] \subset \mathcal{M}_{s}\left(B_{m}\right)$ for every $m>1 / \delta^{2}$. If $\delta<\frac{\epsilon}{2 s}$ and $\left[\delta d_{n}, \epsilon d_{n}\right] \subset \mathcal{M}_{s}\left(B_{m}\right)$, then $\left[\delta d_{n}, \epsilon d_{n}\right] \cap B_{m} \neq \varnothing$. Since this hold for every sufficiently large $m$, we can derive a contradiction as in (i).

The proof of (iii) is the same as proof of (ii).

Proof of Proposition 2.27 We will use the notation from Lemma 2.28. The lemma is trivial if some $c_{k}=\infty$. Thus we will assume that for every $k, c_{k}<\infty$.

Let $k_{1}^{\prime}=1$ and $s_{1}=c_{k_{1}^{\prime}} / 3$. We may choose $k_{1}>k_{1}^{\prime}$ and $b_{1}$ such that $\sup \left\{\frac{b}{a} \mid[a, b] \subset\right.$ $\left.\mathcal{M}_{s_{1}}\left(B_{k}\right)\right\}<b_{1}$ for all $k \geq k_{1}$.

Suppose that we have inductively define $s_{i}, k_{i}^{\prime}, k_{i}$ and $b_{i}$ for all $i<n$.

Choose $k_{n}^{\prime} \in \mathbb{N}$ such that

$$
c_{k_{n}^{\prime}}>b_{n-1}^{3} \cdot c_{k_{n-1}^{\prime}}^{2}
$$

and let $s_{n}=c_{k_{n}^{\prime}} / 3$. Again, we may choose $k_{n}>k_{n}^{\prime}$ and $b_{n}$ such that $\sup \left\{\frac{b}{a} \mid[a, b] \subset\right.$ $\left.\mathcal{M}_{s_{n}}\left(B_{k}\right)\right\}<b_{n}$ for all $k \geq k_{n}$.

Choose $\left[a_{1,1}, b_{1,1}\right]$ a maximal interval in $A_{k_{1}}$ containing a point of $\left(\mathcal{M}_{s_{1}}\left(B_{k_{1}}\right)\right)^{c}$. Suppose that for all $i<n$, we have chosen $\left[a_{i, i}, b_{i, i}\right]$.

Let $\left[a_{n, n}, b_{n, n}\right]$ be a maximal interval in $A_{k_{n}}$ containing a point of $\left(\mathcal{M}_{s_{n}}\left(B_{k_{n}}\right)\right)^{c}$ such that $b_{n-1, n-1}<a_{n, n}$.

Claim Suppose that $x \in\left(\mathcal{M}_{s_{i}}\left(B_{k_{i}}\right)\right)^{c}$. Then $\left[x / s_{i}, x s_{i}\right] \subset A_{k_{i}}$. Additionally, there exist $x^{\prime} \in\left(\mathcal{M}_{s_{i-1}}\left(B_{k_{i-1}}\right)\right)^{c} \cap\left[x / s_{i}, x s_{i}\right]$ such that $\mathcal{M}_{s_{i-1}}\left(\left[x^{\prime} / s_{i-1}, x^{\prime} s_{i-1}\right]\right) \subset$ $\left[x / s_{i}, x s_{i}\right]$. 
Proof of claim Let $x \in\left(\mathcal{M}_{s_{i}}\left(B_{k_{i}}\right)\right)^{c}$. Then $\mathcal{M}_{s_{i}}(x) \cap B_{k_{i}}=\varnothing$ which implies that $\left[x / s_{i}, x s_{i}\right]$ in $A_{k_{i}}$.

Let $a=x / s_{i}$ and $b=x s_{i}$. Then $\frac{x}{a}, \frac{b}{x}=s_{i}=c_{k_{i}^{\prime}} / 3$. This implies that $\frac{b}{a}>\left(c_{k_{i}^{\prime}} / 3\right)^{2}>$ $\left(b_{i-1}^{9} \cdot c_{k_{i-1}^{\prime}}^{4} / 9\right)$.

Let $t=\max \left\{b_{i-1}, c_{k_{i-1}^{\prime}}\right\}$. Since $b /\left(t^{4} a\right)>b_{i-1}$, the interval $\left[a t^{2}, b^{2} / t^{2}\right]$ contains a point $x^{\prime} \in\left(\mathcal{M}_{s_{i-1}}\left(B_{k_{i-1}}\right)\right)^{c}$. Then the inequality $t \geq c_{k_{i-1}^{\prime}}>s_{i-1}$, along with the inclusion $\mathcal{M}_{t}\left(\left[x^{\prime} / t, x^{\prime} t\right]\right) \subset[a, b]$ imply that $\mathcal{M}_{s_{i-1}}\left(\left[x^{\prime} / s_{i-1}, x^{\prime} s_{i-1}\right]\right) \subset[a, b]$. This completes the proof of the claim.

Fix $n$. The claim shows that we can find a nested sequence of intervals $\left[a_{1, n}, b_{1, n}\right] \subset$ $\left[a_{2, n}, b_{2, n}\right] \subset \cdots \subset\left[a_{n, n}, b_{n, n}\right]$ such that $\left[a_{1, n}, b_{1, n}\right] \subset A_{k_{i}}$ and $\mathcal{M}_{s_{i-1}}\left(\left[a_{i, n}, b_{i, n}\right]\right) \subset$ $\left[a_{i+1, n}, b_{i+1, n}\right]$.

Let $d_{n}=\left(a_{1, n} b_{1, n}\right)^{1 / 2}$. Then $\bigcup_{n>i}\left[d_{n} / s_{i}, s_{i} d_{n}\right] \subset A_{k_{n}}$. Therefore $X$ is $\epsilon$-coarsely loop divisible with respect to the pair $(\omega, d)$ for all $\epsilon>0$, since $s_{i}$ diverges.

This gives us the following analogue to Proposition 2.7. Rather than require a bound on the number of pieces in a partition, we only require a linear bound on the diameter of partitions and $\epsilon$-coarsely loop divisible for all $\epsilon>0$.

Proposition 2.29 Suppose that for some fixed pair $(\omega, d)$, a complete geodesic metric space $X$ is $\epsilon$-coarsely loop divisible for all $\epsilon>0$. If there exists an $l, L, N$ and an increasing function $f: \mathbb{N} \rightarrow \mathbb{N}$ such that every loop $\gamma$ in $X$ with $|\gamma| \geq L$ has a partition $\Pi$ of $\gamma$ with the property that

(i) $\Pi$ has at most $f \circ \vartheta^{l}(|\gamma|)$ pieces,

(ii) $\Pi$ is a $\frac{|\gamma|}{2}$-partition of $\gamma$,

(iii) $\operatorname{diam}(\Pi) \leq N|\gamma|$,

then $\operatorname{Con}^{\omega}(X, e, d)$ is simply connected for any choice of observation sequence $e$.

Proof Suppose that for some fixed pair $(\omega, d)$ and all $\epsilon>0, X$ is $\epsilon$-coarsely loop divisible. Fix $l, L, N$ and $f: \mathbb{R} \rightarrow \mathbb{R}$ as in statement of the lemma.

We will break the proof into two parts. First we will show that every geodesic $n-$ gon $\alpha$ in $\operatorname{Con}^{\omega}(X, e, d)$ which is a limit of geodesic $n$-gons from $X$ bounds a disc of diameter at most $2 N|\alpha|$. We will then show that this is enough to imply that all loops are nullhomotopic. 
Step 1 Let $\alpha$ be a geodesic $n$-gon in $\operatorname{Con}^{\omega}(X, e, d)$ such that $\alpha(t)=\left(\alpha_{n}(t)\right)$ where $\alpha_{n}$ is a geodesic $n$-gon in $X$. By hypothesis; for each $n$ such that $\left|\alpha_{n}\right|>L$, there exists a partition $\Pi_{n}$ of $\alpha_{n}$ which satisfy conditions (i)-(iii) of the proposition.

By Lemma 2.3, there exists an integer $K$ and an $\omega$-large set $A$ with the property that $\left(\vartheta^{l}\right)^{-1}\left(\bigcup_{n \in A}\left[|\alpha| d_{n} / 2,2|\alpha| d_{n}\right]\right)$ is bounded by $K$. We will assume that for all $n \in A$, $\left|\alpha_{n}\right| \in \bigcup_{n \in A}\left[|\alpha| d_{n} / 2,2|\alpha| d_{n}\right]$. Thus $\Pi_{n}$ has at most $f(K)$ pieces $\omega$-almost surely.

Then Proposition 2.6 implies that the partitions $\Pi_{n}$ induce a partition $\Pi$ of $\alpha$ which satisfies conditions (i) and (ii) of the proposition. In Papasoglu's proof of Proposition 2.6, $\Pi$ is just the $\omega$-limit of the partitions $\Pi_{n}$; thus, condition (iii) is also satisfied for $\Pi$.

Fix $\gamma$ a geodesic $n$-gon in $\operatorname{Con}^{\omega}(X, e, d)$ such that $\gamma(t)=\left(\gamma_{n}(t)\right)$ for $\gamma_{n}$ a geodesic $n$-gon in $X$.

We have shown that there exists a partition $\Pi_{1}: P_{1}^{(0)} \rightarrow \operatorname{Con}^{\omega}(X, e, d)$ of $\gamma$ into pieces of length $|\gamma| / 2$ with the diameter of the partition no greater than $N|\gamma|$.

Proceeding by induction, suppose that we have defined $\Pi_{k}: P_{k}^{(0)} \rightarrow \operatorname{Con}^{\omega}(X, e, d)$ a partition of $\gamma$ into pieces of length $|\gamma| / 2^{k}$ for $k<i$ such that for all $1<k \leq i-1$

- $\Pi_{k}$ extends $\Pi_{k-1}$,

- for $x \in \operatorname{im} \Pi_{k} \operatorname{dist}\left(x, \operatorname{im} \Pi_{k-1}\right) \leq N|\gamma| / 2^{k}$.

The partition $\Pi_{i-1}$ extends to a map $\widetilde{\Pi}_{i-1}$ on the (1)-skeleton of $P_{i-1}$ as in Remark 1.5. Then we can partition each of the subloops into pieces of length less than $|\gamma| / 2^{i}$ with the desired diameters. We can then use these partitions to extend $\Pi_{i-1}$ to $\Pi_{i}$ satisfying the two induction hypothesis.

For all $i>j$, if $x \in \operatorname{im} \Pi_{i}$, then $\operatorname{dist}\left(x, \operatorname{im} \Pi_{j}\right) \leq \sum_{s=j}^{i} N|\gamma| / 2^{s}$. Hence, $\Pi_{i}$ converges to a continuous function from the unit disc into $\operatorname{Con}^{\omega}(X, e, d)$ which extends $\gamma$. Therefore $\gamma$ bounds a disc of diameter $2 N|\gamma|$. This completes Step 1.

Step 2 Let $Q_{n}$ be the convex hull of the regular $2^{n}$-gon inscribed in $S^{1}$, the unit circle in the plane with the standard Euclidean metric. Then $Q_{n}$ has a natural cell decomposition with $2^{n}$ vertices and $2^{n}$ edges and one 2 -cell. Furthermore, we may assume that the 0 -skeleton of $Q_{n}$ form a nested sequence of subsets of $S^{1}$. Let $A_{1}^{2}=Q_{2}$ which has diameter 2. For $n>2, Q_{n} \backslash\left(\operatorname{interior}\left(Q_{n-1}\right)\right)$ is a set of $2^{n}$ triangles with vertices on $S^{1}$ each of which share a unique edge with $Q_{n-1}$ and have diameter less than $\pi / 2^{n-1}$. Let $\left\{A_{i}^{n}\right\}_{i=1}^{2^{n}}$ be this set of triangles. Then $A=\bigcup_{i, n} A_{i}^{n}$ covers the interior of the unit disc and a dense subset of its boundary.

Fix a loop $\gamma: S^{1} \rightarrow \operatorname{Con}^{\omega}(X, e, d)$. 
We may choose a geodesic 4-gon $\gamma_{1}^{2}: \partial A_{2}^{(1)} \rightarrow \operatorname{Con}^{\omega}(X, e, d)$ such that $\left.\gamma_{1}^{2}\right|_{Q_{2} \cap S^{1}}=$ $\left.\gamma\right|_{Q_{2} \cap S^{1}}$ and $\gamma_{1}^{2}$ is the limit of geodesic 4-gons from $X$. We can inductively define geodesic 3-gons $\left\{\gamma_{i}^{n}: \partial A_{i}^{n} \rightarrow \operatorname{Con}^{\omega}(X, e, d)\right\}$ :

(i) $\left.\gamma_{i}^{n}\right|_{\partial A_{i}^{n} \cap Q_{n-1}}=\left.\gamma_{j}^{n-1}\right|_{\partial A_{i}^{n} \cap Q_{n-1}}$ for some $j$

(ii) $\left.\gamma_{i}^{n}\right|_{\partial A_{i}^{n} \cap S^{1}}=\left.\gamma\right|_{\partial A_{i}^{n} \cap S^{1}}$

Using Step 1, we can define maps $\left\{h_{i}^{n}: A_{i}^{n} \rightarrow \operatorname{Con}^{\omega}(X, e, d)\right\}$ such that $h_{i}^{n}$ is a nullhomotopy of $\gamma_{i}^{n}$ and $\operatorname{diam}\left(h_{i}^{n}\right)$ no greater than $2 N \delta_{i}^{n}$, where $\delta_{i}^{n}$ is the sum of the distances between the image of adjacent vertices of $A_{i}^{n}$.

This defines a function $h: A \rightarrow \operatorname{Con}^{\omega}(X, e, d)$ by $h(a)=h_{i}^{n}(a)$ for some $i$ and $n$. This is well defined by condition (i) above. Since $\gamma$ is continuous on a compact set; for ever $\epsilon>0$, there exists a $K$ such that $2 N \delta_{i}^{n}<\epsilon$ for all $n>K$. Thus $h$ is continuous on $A$. By condition (ii) above, $\left.h\right|_{A \cap S^{1}}=\left.\gamma\right|_{A \cap S^{1}}$ which implies that $h$ extends to a nullhomotopy of $\gamma$.

Corollary 2.30 Let $G$ be a group and $S$ a finite generating set for $G$. Suppose that there exists an $l, L, N$ and an increasing function $f: \mathbb{N} \rightarrow \mathbb{N}$ such that every loop $\gamma$ in $\Gamma(G, S)$ with $|\gamma| \geq L$ has a partition $\Pi$ of with the property that

(i) $\Pi$ has at most $f \circ \vartheta^{l}(|\gamma|)$ pieces,

(ii) $\Pi$ is a $\frac{|\gamma|}{2}$-partition of $\gamma$,

(iii) $\operatorname{diam}(\Pi) \leq N|\gamma|$.

Then at least one of the following occurs.

(A) $G$ has an asymptotic cone which is not semilocally simply connected and has an uncountable fundamental group.

(B) Every asymptotic cone of $G$ is locally simply connected and $G$ has an asymptotic cone which is simply connected.

Proof If for some ultrafilter and scaling sequence $G$ is not $\epsilon$-coarsely divisible for every $\epsilon>0$, then $G$ has an asymptotic cone which is not semilocally simply connected and has uncountable fundamental group.

Otherwise, for every pair $(\omega, d) ; G$ is $\epsilon$-coarsely divisible with respect to $(\omega, d)$ for some $\epsilon>0$. The proof of Proposition 2.29 implies that every asymptotic cone of $G$ is locally simply connected. Proposition 2.27 implies that there exists a pair $(\omega, d)$ such that $G$ is $\epsilon$-coarsely divisible for every $\epsilon>0$ with respect to $(\omega, d)$. Proposition 2.10 implies that $\operatorname{Con}^{\omega}(G, d)$ is simply connected. 


\section{Examples}

Lemma 3.1 Suppose that $G$ is a group with a finite presentation $\langle S \mid R\rangle$ which has an exponential isoperimetric function. If there exists a sequence of loops $\gamma_{n}$ in $\Gamma(G, S)$ such that $\left|\gamma_{n}\right|$ grows at most linearly and $\operatorname{Area}\left(\gamma_{n}\right)$ has an exponential lower bound, then there exists a sequence of absolutely nondivisible loops in $\Gamma(G, S)$.

The hypothesis that the group has a sequence of loops whose areas grow exponential but whose lengths grow linearly is stronger than just having an exponential Dehn function. However, in most known cases this is what is exhibited when showing a group has exponential Dehn function.

Proof Let $\gamma_{n}$ be a sequence of loops in $\Gamma(G, S)$ such that $\left|\gamma_{n}\right| \leq L n$ and $A b^{n} \leq$ $\operatorname{Area}\left(\gamma_{n}\right)=\delta\left(\left|\gamma_{n}\right|\right) \leq D c^{L n}$ where $\delta$ is the Dehn function for the presentation $\langle S \mid R\rangle$ and $A, b, c, D, L$ are positive constants. Fix $M$ such that $c^{L / M} \leq b$.

Suppose that for some subsequence $n_{i}, \gamma_{n_{i}}$ has a $\left|\gamma_{n_{i}}\right| / M$-partition with at most $K$ pieces where $K$ is independent of $i$. Then

$$
A b^{n_{i}} \leq \operatorname{Area}\left(\gamma_{n_{i}}\right)=\delta\left(\left|\gamma_{n_{i}}\right|\right) \leq K \delta\left(\frac{\left|\gamma_{n_{i}}\right|}{M}\right) \leq K D c^{L n_{i} / M} .
$$

This implies that $b^{n_{i}} /\left(c^{L n_{i} / M}\right)=\left(b /\left(c^{L / M}\right)\right)^{n_{i}}$ is bounded independent of $i$ which contradicts our choice of $M$.

Thus $P\left(\gamma_{n},\left|\gamma_{n}\right| / M\right)$ diverges and the lemma follows from Remark 2.23.

Corollary 3.2 Let $G$ be a group from the following list. Then for every ultrafilter $\omega$ and scaling sequence $d, \operatorname{Con}^{\omega}(G, d)$ is not semilocally simply connected and has an uncountable fundamental group which is not free and not simple. In addition, any decomposition of the fundamental group of $\operatorname{Con}^{\omega}(G, d)$ as a free product has a factor which is not free and uncountable.

(1) $\mathrm{SL}_{3}(\mathbb{Z})$

(2) Baumslag-Solitar groups: $B S_{p q}=\left\langle a, t \mid t^{-1} a^{p} t=a^{q}\right\rangle$ for $p, q \neq 0$ and $|p| \neq|q|$

(3) The 3-manifold $\mathrm{Sol}_{3}: \mathbb{R}^{3}$ endowed with the Riemannian metric $d s^{2}=e^{2 z} d x^{2}+$ $e^{-2 z} d y^{2}+d z^{2}$

(4) Any extension of $\mathbb{R}^{n}$ by $\mathbb{R}$ via a matrix with all real eigenvalues of norm strictly greater than 1 and at least two eigenvalues with different sign

(5) Baumslag-Gertsen group: $\left\langle a, t \mid\left(t^{-1} a^{-1} t\right) a\left(t^{-1} a t\right)=a^{2}\right\rangle$ 
(6) $\operatorname{Out}\left(F_{n}\right)$ and $\operatorname{Aut}\left(F_{n}\right)$ for $n \geq 3$

(7) $G_{(7)}=\left\langle a, s, t \mid\left[a, a^{t}\right]=[s, t]=1, a a^{t}=a^{s}\right\rangle$

(8) $G_{(8)}=\left\langle\theta_{1}, \theta_{2}, a, k \mid a^{\theta_{i}}=a, k^{\theta_{i}}=k a, i=1,2\right\rangle$

Recall that the group $G_{(7)}$ is of interest since it is metabelian and not polycyclic. It is sometimes referred to as the Baumslag group. $G_{(8)}$ was constructed by Olshanskii and Sapir and has cubic Dehn function and linear isodiametric function.

Proof Epstein and Thurston in [10] showed the existence of a sequence of loops in $\mathrm{SL}_{3}(\mathbb{R})$ and $B S_{p q}$ for $|p| \neq|q|$ satisfying the conditions of Lemma 3.1.

The result for Baumslag-Solitar groups and $\mathrm{Sol}_{3}$ was already known and is due to Burillo [3]. Cornulier showed the existence of a sequence of absolutely nondivisible loops for groups of the type (4) in [6].

Kassabov and Riley in [17] showed that the loops in the Cayley graph of $G_{(7)}$ with label $\left[a, a^{t^{n}}\right]$ have the desired properties.

For $\operatorname{Out}\left(F_{n}\right)$ and $\operatorname{Aut}\left(F_{n}\right)$ Bridson and Vogtmann exhibit the necessary sequence in [2].

That leaves only (5) and (8). Since $G_{(5)}=\left\langle a, t \mid\left(t^{-1} a^{-1} t\right) a\left(t^{-1} a t\right)=a^{2}\right\rangle$ has a Dehn function which is greater than any tower of exponentials, we cannot apply Lemma 3.1. Gersten in [12] showed the existence of a sequence of loops $\gamma_{k}$ such that $\gamma_{k}$ has length $3 \cdot 2^{k+1}$ and area at least

$$
2^{\left.2^{2^{2}}\right\} k \text { times }}
$$

Platonov in [24] showed that

$$
\delta(n)=2^{\left.2^{2 \cdots 2}\right\} \log _{2}(n) \text { times }}
$$

is an isoperimetric function for $G_{(5)}$.

Suppose that for some subsequence $n_{i}, P\left(\gamma_{n_{i}},\left|\gamma_{n_{i}}\right| / 6\right) \leq K$. Then for all $n_{i}$

$$
2^{\left.2^{2 \cdots{ }^{2}}\right\} n_{i} \text { times }} \leq \operatorname{Area}\left(\gamma_{n_{i}}\right) \leq K 2^{\left.2^{2^{\cdots 2}}\right\} \log _{2}\left(32^{n_{i}} / 6\right) \text { times }}=K 2^{\left.2^{2^{\cdots 2}}\right\}\left(n_{i}-1\right) \text { times }}
$$

which is a contradiction. Hence $\gamma_{k}$ is an absolutely nondivisible sequence of loops and the result follows from Corollary 2.26.

Olshanskii and Sapir in [21] constructed a sequence of loops $\gamma_{n}$ in the Cayley complex of $G_{(8)}=\left\langle\theta_{1}, \theta_{2}, a, k \mid a^{\theta_{i}}=a, k^{\theta_{i}}=k a, i=1,2\right\rangle$ such that $\gamma_{n}$ has length $6 n$. 
Additionally, they showed that $\gamma_{n}$ cannot bound a disc decomposed into at most $l$ subdiscs of perimeter $n$ where $l \leq \sqrt{n}$ and hence is an absolutely nondivisible sequence of loops.

Remark 3.3 Suppose that $X$ is a one-dimensional metric space and $Y$ is the support of any finite set of paths in $X$. Then $Y$ is a compact one-dimensional metric space and thus its fundamental group is locally free, residually free and residually finite by Cannon and Conner [5, Theorem 5.11]. Since $X$ is one-dimensional, the homomorphism from the fundamental group of $Y$ to the fundamental group of $X$ induced by set inclusion is injective; see [5, Theorem 3.7]. Thus $\pi_{1}\left(X, x_{0}\right)$ is locally free.

Burillo in [3] shows that all asymptotic cones of solvable $B S_{p, q}$ with $|p| \neq|q|$ and $\mathrm{Sol}_{3}$ have topological dimension 1 . As well, any extension of $\mathbb{R}^{n}$ by $\mathbb{R}$ via a matrix with all real eigenvalues of norm strictly greater than 1 and at least two eigenvalues with different sign will have one-dimensional asymptotic cones; see [6]. Thus the fundamental groups of their cones are locally free.

To prove Corollary 3.2, we analysed mappings of Hawaiian earrings into asymptotic cones and showed that the induced homomorphism's image had the desired properties. However, this method doesn't give us much information concerning the structure of the rest of the fundamental group. When an asymptotic cones of a group is one-dimensional, one can apply standard techniques for one-dimensional space, as in Remark 3.3, to better understand the structure of the fundamental. Requiring a dimension constraint on the asymptotic cone is a strong condition and does not apply to many well studied groups.

\subsection{An example of a group with locally simply connected cones which is not simply connected}

In [19], Olshanskii showed that there exists a group with the following properties.

Theorem 3.4 There is a finitely generated group $G$ whose Dehn function $f(n)$ satisfies the following properties:

(1) There are sequences of positive numbers $d_{i} \rightarrow \infty$ and $\lambda_{i} \rightarrow \infty$ such that $f(n) \leq c n^{2}$ for arbitrary integer $n \in\left[d_{i} / \lambda_{i}, d_{i} \lambda_{i}\right]$ and some constant $c$.

(2) There is a positive constant $c^{\prime}$ and an increasing sequence of numbers $n_{i} \rightarrow \infty$ such that $f\left(n_{i}\right) / n_{i}^{2} \rightarrow \infty$ but for every $i$, and for every integer $n$ with $n<c^{\prime} n_{i}$, we have $f(n) \leq c^{\prime} n_{i}^{2}$. 
Olshanskii constructed $G$ as a multiple HNN extension of a free group using $S-$ machines.

Corollary 3.5 (A) There exists an $\omega$ such that $\operatorname{Con}^{\omega}\left(G,\left(n_{i}\right)\right)$ has a nontrivial fundamental group.

(B) $\operatorname{Con}^{\omega}\left(G,\left(n_{i}\right)\right)$ is locally simply connected for all $\omega$.

(C) $\operatorname{Con}^{\omega}\left(G,\left(d_{i}\right)\right)$ has trivial fundamental group for all $\omega$.

Proof Olshanskii and Sapir in [22] showed that the second condition implies the existence of a $b<1$ such that $f\left(n_{i}\right) / f\left(b n_{i}\right) \rightarrow \infty$. This was used to show that divisibility function restricted to $\bigcup_{i}\left[b n_{i}, n_{i}\right]$ is unbounded. Then (A) follows.

The first condition implies that $G$ is uniformly $\epsilon$-coarsely loop divisible for every $\epsilon>0$ with respect to the pair $\left(\omega,\left(d_{i}\right)\right)$ for any ultrafilter $\omega$. Therefore $\operatorname{Con}^{\omega}\left(G,\left(d_{i}\right)\right)$ has trivial fundamental group.

The second condition implies (by the same argument that was used to show $G$ is uniformly $\epsilon$-coarsely loop divisible for every $\epsilon>0$ with respect to the pair $\left.\left(\omega,\left(d_{i}\right)\right)\right)$ that there exists an $\epsilon>0$ such that $G$ is uniformly $\epsilon$-coarsely loop divisible for the pair $\left(\omega,\left(n_{i}\right)\right)$. Hence, $\operatorname{Con}^{\omega}\left(G,\left(n_{i}\right)\right)$ is locally simply connected.

Question 4 Can this group have an asymptotic cone which is not locally simply connected?

Or for finitely presented groups in general we can ask the following:

Question 5 Is there a finitely presented group which has a locally simply connected asymptotic cone and an asymptotic cone which is not locally simply connected?

Question 5 is false if we consider the larger class of finitely generated groups. Thomas and Velicovick consider a group $G_{I}=\left\langle a, b \mid\left(a^{n} b^{n}\right)^{7}=1 ; n \in I\right\rangle$ which they show for an appropriate choice of $I$ has a simply connected and nonsimply connected asymptotic cone; see [28].

Proposition 3.6 Let $I_{0}=\left\{2^{2^{n}}\right\}$. Let $I_{0, k}=I_{0} \cap\left[2^{2^{k}}, \infty\right)$ and $I=\bigcup_{k=1}^{\infty} 2^{k} \cdot I_{0, k}$. Then $G_{I}=\left\langle a, b \mid\left(a^{n} b^{n}\right)^{7}=1 ; n \in I\right\rangle$ has a cone which is locally simply connected and a cone which is not semilocally simply connected. 
Proof Let $\gamma_{n}$ be the loop based at the identity with label $\left(a^{n} b^{n}\right)^{7}$ for $n \in I$. Then Thomas and Velicovick show using small cancellation that $P\left(\gamma_{n},\left|\gamma_{n}\right| / 2\right)=\infty$; see [28, Lemma 1.1].

If we let $d_{n}=8^{2^{n-1}}$, then the argument of Thomas and Velicovick shows that $\operatorname{Con}^{\omega}(G, d)$ is an $\mathbb{R}$-tree for any $\omega$.

Let $\rho_{n}=2^{n} 2^{2^{n}}=2^{2^{n}+n}$. Let $\gamma_{n, k}$ be the loop with label $\left(a^{2^{2^{n}+k}} b^{2^{2^{n}+k}}\right)^{7}$ for $k \geq n$. Then $\left|\gamma_{n, k}\right| / \rho_{n}=14 / 2^{n-k}$. Hence $\left(\gamma_{n, n-i}\right)$ is a loop of length $14 / 2^{i}$ in $\operatorname{Con}^{\omega}\left(G,\left(\rho_{n}\right)\right)$ which has no finite partition. Hence $\operatorname{Con}^{\omega}\left(G,\left(\rho_{n}\right)\right)$ is not semilocally simply connected and has uncountable fundamental group for any $\omega$.

Acknowledgements The authors would like to thank Mark Sapir for may helpful discussions and suggestions with the writing of this paper. As well, we would like to thank Yves Cornulier for pointing out that the cones of solvable Baumslag-Solitar groups, $\mathrm{Sol}_{3}$ and some extensions of $\mathbb{R}^{n}$ by $\mathbb{R}$ are bilipschitz.

\section{References}

[1] M R Bridson, Asymptotic cones and polynomial isoperimetric inequalities, Topology 38 (1999) 543-554 MR1670404

[2] M R Bridson, K Vogtmann, The Dehn functions of $\operatorname{Out}\left(F_{n}\right)$ and $\operatorname{Aut}\left(F_{n}\right)$, Ann. Inst. Fourier (Grenoble) 62 (2012) 1811-1817 MR3025154

[3] J Burillo, Dimension and fundamental groups of asymptotic cones, J. London Math. Soc. 59 (1999) 557-572 MR1709665

[4] J W Cannon, G R Conner, The combinatorial structure of the Hawaiian earring group, Topology Appl. 106 (2000) 225-271 MR1775709

[5] J W Cannon, G R Conner, On the fundamental groups of one-dimensional spaces, Topology Appl. 153 (2006) 2648-2672 MR2243740

[6] Y de Cornulier, Dimension of asymptotic cones of Lie groups, J. Topol. 1 (2008) 342-361 MR2399134

[7] C Druţu, Quasi-isometry invariants and asymptotic cones, Internat. J. Algebra Comput. 12 (2002) 99-135 MR1902363

[8] C Druțu, M V Sapir, Tree-graded spaces and asymptotic cones of groups, Topology 44 (2005) 959-1058 MR2153979

[9] K Eda, Atomic property of the fundamental groups of the Hawaiian earring and wild locally path-connected spaces, J. Math. Soc. Japan 63 (2011) 769-787 MR2836742 
[10] D B A Epstein, J W Cannon, D F Holt, S V F Levy, M S Paterson, W P Thurston, Word processing in groups, Jones and Bartlett Publishers, Boston, MA (1992) MR1161694

[11] A Erschler, D V Osin, Fundamental groups of asymptotic cones, Topology 44 (2005) 827-843 MR2136537

[12] S M Gersten, Dehn functions and $l_{1}-$ norms of finite presentations, from: "Algorithms and classification in combinatorial group theory", (G Baumslag, editor), Math. Sci. Res. Inst. Publ. 23, Springer, New York (1992) 195-224 MR1230635

[13] M Gromov, Hyperbolic groups, from: "Essays in group theory", (S M Gersten, editor), Math. Sci. Res. Inst. Publ. 8, Springer, New York (1987) 75-263 MR919829

[14] M Gromov, Asymptotic invariants of infinite groups, from: "Geometric group theory, Vol. 2”, (G A Niblo, M A Roller, editors), London Math. Soc. Lecture Note Ser. 182, Cambridge Univ. Press (1993) 1-295 MR1253544

[15] A Hatcher, Algebraic topology, Cambridge Univ. Press (2002) MR1867354

[16] G Higman, Unrestricted free products, and varieties of topological groups, J. London Math. Soc. 27 (1952) 73-81 MR0045730

[17] M Kassabov, T R Riley, The Dehn function of Baumslag's metabelian group arXiv : 1008.1966

[18] C Kent, Asymptotic cones of HNN-extensions and amalgamated products

[19] A Y Olshanskii, Groups with quadratic-non-quadratic Dehn functions, Internat. J. Algebra Comput. 17 (2007) 401-419 MR2310153

[20] A Y Olshanskii, D V Osin, M V Sapir, Lacunary hyperbolic groups, Geom. Topol. 13 (2009) 2051-2140 MR2507115

[21] A Y Olshanskii, M V Sapir, Groups with non-simply connected asymptotic cones, from: "Topological and asymptotic aspects of group theory", (R Grigorchuk, M Mihalik, M V Sapir, Z Šunik, editors), Contemp. Math. 394, Amer. Math. Soc. (2006) 203-208 MR2216717

[22] A Y Olshanskii, M V Sapir, A finitely presented group with two non-homeomorphic asymptotic cones, Internat. J. Algebra Comput. 17 (2007) 421-426 MR2310154

[23] P Papasoglu, On the asymptotic cone of groups satisfying a quadratic isoperimetric inequality, J. Differential Geom. 44 (1996) 789-806 MR1438192

[24] A N Platonov, An isoparametric function of the Baumslag-Gersten group, Vestnik Moskov. Univ. Ser. I Mat. Mekh. (2004) 12-17, 70 MR2127449 In Russian; translated in Moscow Univ. Math. Bull. 59 (2004) 12-17

[25] T R Riley, Higher connectedness of asymptotic cones, Topology 42 (2003) 1289-1352 MR1981358 
[26] M V Sapir, J-C Birget, E Rips, Isoperimetric and isodiametric functions of groups, Ann. of Math. 156 (2002) 345-466 MR1933723

[27] E Summers, There is no surjective map from the Hawaiian earring group to the double Hawaiian earring group, master's thesis, Brigham Young University (2002)

[28] S Thomas, B Velickovic, Asymptotic cones of finitely generated groups, Bull. London Math. Soc. 32 (2000) 203-208 MR1734187

Department of Mathematics, Brigham Young University 275 TMCB, Provo, UT 84602, USA

Mathematics Department, University of Toronto 40 St. George Street, Room 6290, Toronto ON M5S 2E4, Canada

conner@math.byu.edu, curt.kent@utoronto.ca www.math.byu.edu/ conner/, http://www.math.toronto.edu/cms/kent-curt/

Received: 16 October 2012 Revised: 1 August 2013 
